\title{
POST-DISASTER RECOLONIZATION OF MANGROVE FORESTS WITH A STOCHASTIC AGENT-BASED MODEL
}

\author{
V. P. Bongolan ${ }^{\mathrm{a}}$, J. E. Branzuela ${ }^{\mathrm{a}}$, G. M. Torres ${ }^{\mathrm{a}}$ \\ ${ }^{\text {a }}$ Scientific Computing Laboratory, Department of Computer Science, University of the Philippines Diliman
}

KEY WORDS: Mangroves, Mangrove Soil Properties, Recolonization, Mangrove Simulation, Agent-based Modelling

\begin{abstract}
:
Mangrove forests in the Philippine coastline are susceptible to severe damage due to tropical storms. These mangrove forests provide a home for other plants and animals as well as providing resources for people living in coastal areas. Thus, it is important to promote proper conservation and judicious replanting in areas affected by storms. Since different species vary on their tolerance to physical conditions such as water salinity and soil composition, the appropriate genus must be used in reforestation efforts. This study aims to model the change in soil composition due to the introduction of a non-native species, Rhizophora mucronata, and restoring soil condition to aid recolonization of the existing native species, Avicennia and Sonneratia.

The study uses an agent-based model for the prediction of the regenerative behaviour of mangrove stands consisting of the native species and the planted or non-native species in a fragmented habitat, with the use of spatio-temporal coloured noise to simulate stochastic seedling dispersal and subject to storm damage. The model uses Salmo and Juanico's model for mangrove growth. Stochastic experiments were carried out in a shoreline habitat with an existing native population of varying ages and a larger population of planted, non-native seedlings. The GIS data of Bangrin Marine Protected Area was used to simulate the recovery trajectory of the stand after typhoon Chan-hom of 2009.
\end{abstract}

\section{INTRODUCTION}

\subsection{Background}

Mangrove trees and shrubs are a common sight on mudflats and banks of tropical and subtropical rivers and coastlines in many parts of the world, standing with their roots in salt water (Spalding et al., 2010). They exist in conditions of high salinity, extreme tides, strong winds, high temperatures and muddy, anaerobic soils.Mangroves provide habitat to various organisms and are used to supply building material, firewood, charcoal, food and medicine. They are also known for inducing sedimentation and performing carbon sequestration (Kandasamy and Bingham, 2001), accounting for $14 \%$ of carbon sequestration by coastal forests (Alongi, 2012).

Mangroves exhibit vivipary; their seeds grow while still attached to the tree. Mangrove seeds detach from their parents and disperse until they find stable ground. Some mangroves have been known to live for more than 100 years. However, many mangrove stands in the Philippines never reach full maturity because of frequent, devastating storms (Bongolan et al., 2017). Additionally, human activities such as logging and urban development contributes to the destruction of mangrove forests. The habitat loss can be traced back as early as the 1950s where vast tracts of mangrove areas were logged for firewood and tannins and further exacerbated in the 1960s when government adopted a policy aimed at increasing fish production by converting large areas of mangroves into culture ponds (Barnuevo et al., 2017).

Efforts of mangrove conservation and reforestation met with mixed results. These efforts were often unsuccessful, largely because of the high mortality of the seedlings, inappropriate site selection, predation, over topping by waves and barnacle infestation. Most of the rehabilitation programs utilized only single species of Rhizophora, creating a mono specific plantation with no post-planting management plan. In the work of Barnuevo et al. (Barnuevo et al., 2017), they have observed that the secondary succession of densely planted Rhizophora in Banacon Island was inhibited as reflected by the very low percentage of regeneration potential. Meanwhile, recruitment and colonization of nonplanted species was promoted in Olango Island.

There are a number of factors affecting regeneration due to difference in adaptation between species. In Hesse's work (Hesse, 1961), it is observed that soil found in Rhizophora forests have higher density of sulphide than Avicennia. Our study aims to simulate mangrove recolonization with the focus of factoring soil conditions effect on it.

\subsection{Importance of Mangroves}

Mangroves are woody plants that grow between land and sea in tropical and subtropical latitudes. Each mangrove tree is a biome, and forms an ecosystem with various bacteria, fungi and other fauna and flora. Organisms such as algae, sponges, nematodes, sea marsh plants, fish, shrimp, crabs, dragonflies, bees and ants can be found in mangrove forests (Kandasamy and Bingham, 2001). These forests also offer shoreline protection, carbon sequestration and nutrient regulation (Lee et al., 2014).

Mangroves are critically endangered in 26 out of 120 countries hosting them. Coastal woodlands are rapidly disappearing in developing countries which houses $90 \%$ of mangroves. In a study by Richards and Friess (Richards and Friess, 2016), there was a loss of mangrove forests at an average rate of $0.18 \%$ per year in Southeast Asia between 2000 and 2012. A total of 100,000 hectares of mangroves were gone during the period. The decline endangers a large number of organisms relying on mangrove forests for their survival.

\subsection{Mangrove Restoration}

In 2009, Typhoon "Chan-hom" inflicted heavy damage in the coastal woodlands of Lingayen Gulf located in the Philippines. Damage was spread across mangrove stands, with stands 11 and 18 years old damaged the most. Field observations suggests that taller trees have higher mortality rate whenever a storm hits, as 
compared to smaller trees. This may be the reason for the low biomass reported for mangrove forests in the Philippines. Since the country has a high frequency of storms, older and taller mangroves are destroyed more often (Salmo et al., 2013b) in the stands within a destructive storms path.

\subsection{Mangrove Soil Properties}

Due to harsh conditions, mangroves have evolved to suit their environments. There is a significant amount of genetic variability between mangrove species (Kandasamy and Bingham, 2001). Different species dominate certain areas due to differences in rooting and geographic conditions such as inundation and salinity (Aksornkoae et al., 1993).

Soil properties have been observed by several studies (Boto and Wellington, 1984), (Reef et al., 2010), (McKee, 1993), (Salmo et al., 2013a) which vary between species. Hesse (Hesse, 1961) reports that soil associated with Rhizophora has a higher $\mathrm{pH}$ value, sulphur, nitrogen and phosphorus. Fibrous mud under Rhizophora trees contained over six times as much monosulfide compared to non-fibrous mud under Avicennia trees. In the study of Lyimo and Mushi (Lyimo and Mushi, 2005), their experiment showed that seedling establishment did not occur with high sulfide concentration $(0.5-0.6 \mathrm{mM})$ and low redox potential (-27 to $-198 \mathrm{mV}$ ). However, mangrove trees allows aeration which reduces sulfide concentrations and helping the growth of mangrove seedlings. Changes in soil properties particularly an increase in soil nutrients, reduced organic matter, more anoxic soils and increased salinity can also be observed after a disaster occurs (Salmo et al., 2013b).

To counter the increase of sulfur in soil, an aqueous calcium hydroxide solution can be sprayed or applied to sop-up the excess sulfur into calcium sulphate. The reaction should happen in the wild, with no further human intervention. Below is the stoichiometry of calcium hydroxide plus sulfate which will result in calcium sulfate plus two hydroxide ions.

$$
\mathrm{Ca}\left(\mathrm{OH}_{2}\right)+\mathrm{SO}_{4}^{2-}->\mathrm{CaSO}_{4}+2\left(\mathrm{OH}^{-}\right)
$$

This can be tried in experimental plots. Please note that calcium sulfate is still water soluble, although it is inert. Further, the hydroxide ions could change the soil $\mathrm{pH}$, being a base.

\section{MODEL OVERVIEW, DESIGN CONCEPTS AND DETAILS}

The simulation is based on work by Velasco et al. (Bongolan et al., 2017) where they calibrated the stochastic model by Fiestada and Lorbis (Fiestada and Lorbis, 2017). The work uses an agent-based implementation by Ang and Mariano (Ang and Mariano, 2016) based on an existing mathematical model of mangrove growth by Salmo and Juanico (Salmo and Juanico, 2015). Stochasticity was added by generating spatiotemporal colored noise for seedling dispersal; spatial inheritance is simulated by a variance in allometric attributes of individual mangrove agents. The model is implemented in NetLogo and allows for a high level of customization. Other improvements include differentiation among the recruitment of viviparous genera and increased resistance.

The model aims to be a generalized model of growth or regeneration of a hetero-specific mangrove forest in a fragmented habitat. In particular, the Avicennia, Sonneratia (native to the areas) and Rhizophora (planted in the areas) genera are investigated in a virtual, analog of the Bangrin Marine Protected Area in Bani, Pangasinan.

\subsection{Agents}

The main agent being modeled is the mangrove plant. These mangrove agents have a single state variable that determines their growth and maturity: the diameter at breast height of the mangrove, which we will refer to as $D$. Empirical data can be used to allometrically relate $D$ to other factors such as tree height and weight (Hiebeler, 2000).

2.1.1 Agent Classification Agents can be classified in two ways: by genus and by maturity. As the model deals with heterospecific forests, the behavior of mangrove agents is modified by genus-specific parameters. Mangroves are also divided into maturity levels. In Ang and Mariano (Ang and Mariano, 2016), there were three maturity levels (dependent on $D$ ) ranging from seedling to sapling to tree, with different mortality rates tied to each maturity level per genus. The genus-specific mortality rates were modified in Velasco et al. (Bongolan et al., 2017). The chance that a tree will die from natural causes is now tied solely to its D (diameter at breast height). In Ang and Mariano (Ang and Mariano, 2016), Sonneratia and Avicennia trees (with $D \geq 5 \mathrm{~cm}$ ) had a 0.166 chance of dying from natural causes; for saplings $(D \in[2.5,5])$, it was 0.2 , and for seedlings $(D \in[0.5,2.5))$, it was 0.4 . Rhizophora had slightly higher mortality rates for each maturity level. The same general trend of higher mortality for less mature plants was followed all genera. In the present model, a Lagrange interpolated mortality rate is obtained from these values, so that a plant's chances of dying from natural causes gradually but steadily declines as its $D$ increases (Bongolan et al., 2017).

\subsection{Spatial Units}

The model simulates mangrove agents in a closed patch grid consisting of some land area and sea area, separated by an arbitrarily shaped coast. Some patches are considered as non-livable (e.g. patches that represent the sea or land used by humans). The site was divided into $350 \times 350$ patches where each patch corresponds to the real-world area roughly $2.14 \mathrm{~m} \times 2.14 \mathrm{~m}$ in size. (Bongolan et al., 2017).

2.2.1 Coastal Distance Salinity and tidal inundation responses are approximately dependent on distance from the coast. The farther from the coast a patch is, the less inundated and the less saline it should be due to having less exposure to seawater. Each patch thus has a specific inundation and salinity effect on the plants in it (Bongolan et al., 2017).

\subsection{Variables}

The model's determination of individual mangrove growth follows the differential equation proposed by Salmo and Juanico (Salmo and Juanico, 2015). The deterministic change in D with respect to time is as follows:

$$
\begin{aligned}
\frac{d D}{d t}= & \left(\frac{\Omega}{2+\alpha}\right) D^{\beta-\alpha-1}\left[1-\frac{1}{\gamma}\left(\frac{D}{D_{\max }}\right)^{1+\alpha}\right] \\
& \times \sigma(x, y) \times \eta(x, y) \times K(x, y)
\end{aligned}
$$

where $\quad \alpha=$ allometric constant relating $D$ to tree height $\beta=$ allometric constant relating $D$ to crown radius $\Omega=$ conversion factor that harmonizes both sides of the equation $\gamma=$ genus-specific constant related to maximum height 


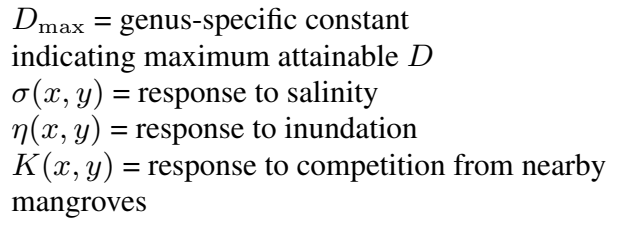

Salmo and Juanico also provide formulae for calculating the responses to salinity, tidal inundation and competition from patch values and neighborhood characteristics.

The allometric parameters, $\alpha$ and $\beta$ will be genus-specific but will also vary randomly among individuals of the same genus. The values will be inherited directly by seedlings and will simulate fitness and spatial inheritance, as optimal values for each patch should be selected for. Spatial inheritance is a phenomenon in which genetically related individuals inherit a habitat or continue to thrive in an area for multiple generations. This occurs when organisms do not disperse far and become highly suited to an area (Schauber et al., 2007).

2.3.1 Recruitment Each unoccupied, livable patch has a chance of recruiting a seedling. Initially, the chance of recruitment is zero for all patches. When a mangrove reaches maturity (becomes a tree with a diameter of at least $5 \mathrm{~cm}$ ), the recruitment chance for its patch becomes 0.5 . The recruitment chance for all other patches changes as governed by the following equation (Bongolan et al., 2017).

$$
\frac{d \Pi}{\mathrm{dt}}=\frac{-1}{\tau}\left(1-\lambda^{2} \nabla^{2}\right) \Pi+\frac{1}{\tau} \omega
$$

with

$$
\Pi_{0}=0
$$

Here, $\omega_{i}$ is a white noise term that follows Brownian motion. It can be easily generated independently for each patch for every step of the simulation.

For each patch at a given step $i$,

$$
\omega_{i}=\omega_{i-1}+\operatorname{Normal}(0, d t)
$$

with

$$
\omega_{0}=0
$$

$\nabla^{2} \Pi=\Pi_{\mathrm{xx}}+\Pi_{\mathrm{yy}}$ can be approximated using the finite difference method. For the boundaries, we may assume that recruitment does not occur (this is not such a big problem since the map used is bounded by water and area not part of the coastal woodlands). At step $i$ of the simulation, let the recruitment chance at a livable, unoccupied patch $j, k$ be

$$
\begin{aligned}
\Pi_{j, k}^{i} & =\Pi_{j, k}^{i-1}+d t\left[\left(\frac{-1}{\tau}\right) \Pi_{j, k}^{i-1}\right. \\
& +\left(\frac{\lambda^{2}}{\tau}\right) \frac{\Pi_{j+1, k}^{i-1}-2 \Pi_{j, k}^{i-1}+\Pi_{j-1, k}^{i-1}}{(\Delta x)^{2}} \\
& \left.+\left(\frac{\lambda^{2}}{\tau}\right) \frac{\Pi_{j, k+1}^{i-1}-2 \Pi_{j, k}^{i-1}+\Pi_{j, k-1}^{i-1}}{(\Delta y)^{2}}+\frac{1}{\tau} \omega_{j, k}^{i}\right]
\end{aligned}
$$

The values for the correlation time and length are varied throughout the experiments (Bongolan et al., 2017).

\subsection{Process Overview}

The simulation is initialized by creating the virtual environment (with salinity and inundation values calculated for each patch using their distance from the sea and sulfide content based on Hesse's (Hesse, 1961) work) and planting the initial populations or mangroves. During each step, a Poisson-distributed random time interval with a mean of 1 day is generated as per Salmo and Juanico (Salmo and Juanico, 2015). Each agent/mangrove grows in diameter according to the growth equation and proportionally to $d t$; that is, the change in diameter during a step in the simulation is calculated as $\frac{\mathrm{dD}}{\mathrm{dt}} d t=d D$ (Bongolan et al., 2017).

During the same step, the recruitment chance for each patch is recalculated. A randomly selected mangrove may die from natural causes, with a probability dependent on its maturity. A randomly selected patch may also recruit a new seedling dependent on its recruitment chance (Bongolan et al., 2017).

A Poisson-distributed inter-arrival time for storms is assumed. Each mature tree, irrespective of genus, has a probability of dying equal to $0.7-k n$, where $k$, is a parameter of the simulation and $n$ is the number of neighboring trees within the focal tree's field of neighborhood. Smaller plants have a 0.1 chance of dying (Bongolan et al., 2017).

Patches affected by the storm will have their sulphide content changed to simulate spraying of calcium hydroxide solution on the soil.

\subsection{Design and Concepts}

Storms and death are both modeled as natural Poisson processes with varying rates depending on genus and maturity. Seedling dispersal is modeled as diffusion using spatiotemporal colored noise (Bongolan et al., 2017).

2.5.1 Emergence and Adaptation The model can simulate the emergence of a naturally-selected population of mangroves over several generations, affected by both the fragmentation of the mangrove forest (plants are not uniformly distributed) and the properties of the plants themselves. It will thus be able to take into account the effects of having multiple genera and an arbitrary landscape.

The variations in the properties of each individual agent or plant, as well as the tendency for plants that are more fit to survive longer and have more offspring will simulate spatial selection within the population (Bongolan et al., 2017).

2.5.2 Interaction Plant agents interact with each other by competing for resources, which affects growth, and thus also influences mortality rates. The more neighbors a plant has, the slower it should grow, and thus the greater the chances of it dying during each step. However, having more neighbors also reduces the mortality rate of the tree during storms, since having more neighbors distributes the force and damage inflicted by the storm (Bongolan et al., 2017).

2.5.3 Soil Properties Patches have differing sulphur content depending on the genus of mangrove planted. Initial values are taken from Hesse's (Hesse, 1961) observation on Avicennia and Rhizophora mangroves. Patches destroyed by a storm have their sulphur content changed to simulate soil changes by spraying calcium hydroxide solution. Patches with non-native genus will have reduced sulphide content like patches with native genus. There will be no change in sulphide content on patches with native genus. 
2.5.4 Initialization and Input The forest was initialized with 4000 native mangroves and 10000 planted mangroves of varying maturity levels. On the 1000th day of the simulation, a storm is expected to occur (the estimated return time for the study area for a storm of Chan Homs strength is four or five times a century or 22.5 years). The natives were given $20-40 \%$ more resistance against environmental stressors and storms (Bongolan et al., 2017).

\section{RESULTS AND DISCUSSION}

A stochastic experiment was conducted to simulate the condition of mangrove forest within 100 years. The recovery trajectory of the mangrove forest is determined by its forest cover. The forest cover is the sum of crown areas of all mangroves in a population. Crown area is determined by the plant's diameter at breast height (Berger and Hildenbrandt, 2000), where $r_{\text {crown }}=11.1 D^{0.645}$. The crown area is calculated using the crown radius (in square centimeters) with the assumption that the tree crown is a circular region. Planted mangroves are colored blue-green while native mangroves are colored green. Native mangroves that colonized patches from planted mangroves after a storm are colored red. Experiments are done by varying effectiveness in changing sulphide content, e.g., 30 percent successful, 50 percent successful, etc.

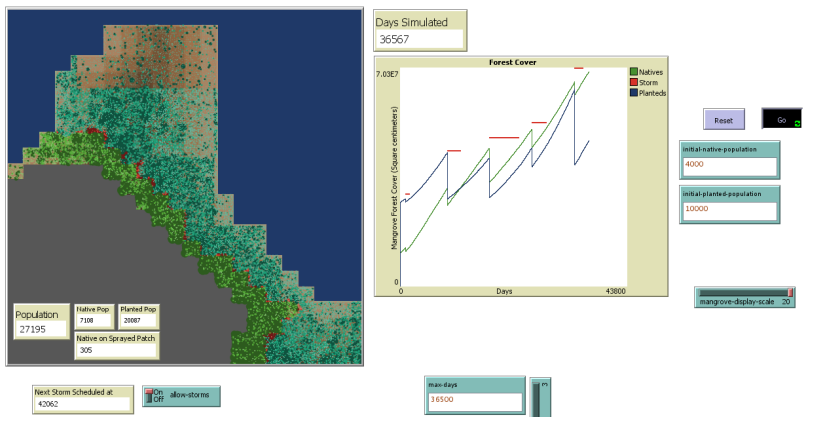

Figure 1: A simulation with a success rate of $30 \%$

On average, sprayed patches assist the growth of native mangroves only when they are near a native mangrove. Although the population of native mangroves in sprayed patches is not big compared to native mangroves in established native patches, they are still successful in colonizing planted patches.

Previous experimental results on post-disaster regeneration remain as is: native mangroves fully recovered in 5-7 years while the planted mangroves recovered only $40 \%$ of its pre-storm cover in 6-8 years. The planted mangroves had an advantage for having a larger population but as the years progress, the population of the mature planted trees decreases; they are simply not suited to the area. On the other hand, the forest cover of native mangroves had increased through the years.

\begin{tabular}{|l|c|c|}
\hline \% Effectivity & Average No. & \% of Native Mangrove \\
\hline 0.30 & 336.00 & 0.0485 \\
0.35 & 460.33 & 0.0639 \\
0.50 & 556.33 & 0.0766 \\
\hline
\end{tabular}

Table 1: Average number of mangroves in sprayed patches

\section{CONCLUSION AND RECOMMENDATIONS}

This study shows the simulation of changing soil properties postdisaster to aid recolonization of native mangrove. Sprayed patches near native mangroves were successfully colonized. In the study by Barnuevo et al. (Barnuevo et al., 2017), they reported that the growth of planted mangrove forests were inhibited reflected by the very low percentage of regeneration potential. In contrast, the recruitment and colonization of native mangroves were promoted. Therefore, it is important to help native mangroves repopulate destroyed coastlines. The researchers recommend further study on the changes and effect of soil composition between mangrove genera to help decision making in conservation and restoration. Post-planting management should be practiced to monitor the regeneration of the forest and to gather data for future studies.

\section{ACKNOWLEDGEMENTS}

The authors would like to express our sincere gratitude to Vena Pearl A. Bongolan Ph.D. for guiding and giving feedback on our work. We would also like to thank Ariel C. Blanco Ph.D., Engr. Edgardo G. Macatulad and the rest of IAMBlueCECAM for allowing us to attend the National Blue Carbon Symposium and answering our inquiries regarding our works.

\section{REFERENCES}

Aksornkoae, S. et al., 1993. Ecology and management of mangroves. Technical report, Kasetsart University.

Alongi, D. M., 2012. Carbon sequestration in mangrove forests. Carbon Management 3(3), pp. 313-322.

Ang, F. D. and Mariano, S. J., 2016. An agent-based model of post-disaster mangrove forest regrowth. Technical report, University of the Philippines Diliman.

Barnuevo, A., Asaeda, T., Sanjaya, K., Kanesaka, Y. and Fortes, M., 2017. Drawbacks of mangrove rehabilitation schemes: Lessons learned from the large-scale mangrove plantations. Estuarine, Coastal and Shelf Science.

Berger, U. and Hildenbrandt, H., 2000. A new approach to spatially explicit modelling of forest dynamics: Spacing, ageing and neighbourhood competition of mangrove trees. Ecological Modelling 132, pp. 287302

Bongolan, V. P., Salmo, S. G. and Velasco, L. A., 2017. A stochastic agent-based model for mangrove forest regrowth. Technical report, University of the Philippines Diliman.

Boto, K. and Wellington, J., 1984. Soil characteristics and nutrient status in a northern australian mangrove forest. Estuaries 7, pp. 61-69.

Fiestada, V. and Lorbis, A., 2017. A stochastic agent-based model for mangrove forest regrowth. Technical report, University of the Philippines Diliman.

Hesse, R. P., 1961. Some differences between the soils of rhizophora andavicennia mangrove swamps in sierra leone. Plant and Soil 14, pp. 335-346.

Hiebeler, D., 2000. Populations on fragmented landscapes with spatially structured heterogeneities: Landscape generation and local dispersal. Ecology 81(6), pp. 1629-1641.

Kandasamy, K. and Bingham, B., 2001. Biology of mangroves and mangrove ecosystems. Advances in Marine Biology 40, pp. 81-251. 
Lee, S., Primavera, J., Dahdouh-Guebas, F., McKee, K., Bosire, J., Cannicci, S., Diele, K., Fromard, F., Koedam, N., Marchand, C., Mendelssohn, I., Mukherjee, N. and Record, S., 2014. Ecological role and services of tropical mangrove ecosystems: A reassessment. Global Ecology and Biogeography.

Lyimo, T. and Mushi, D., 2005. Sulfide concentration and redox potential patterns in mangrove forests of dar es salaam: Effects on avicennia marina and rhizophora mucronata seedling establishment. Western Indian Ocean Journal of Marine Sciences 4, pp. 163-173.

McKee, K., 1993. Soil physicochemical patterns and mangrove species distribution-reciprocal effects? Journal of Ecology 81(3), pp. 477-487.

Reef, R., Feller, I. and Lovelock, C., 2010. Nutrition of mangroves. Tree physiology 30, pp. 1148-60.

Richards, D. and Friess, D., 2016. Rates and drivers of mangrove deforestation in southeast asia, 2000-2012. Proceedings of the National Academy of Sciences 113(2), pp. 344-349.

Salmo, S. and Juanico, D. E., 2015. An individual-based model of long-term forest growth and carbon sequestration in planted mangroves under salinity and inundation stresses. International Journal of Philippine Science and Technology 8(2), pp. 31-35.

Salmo, S., Lovelock, C. and Duke, N., 2013a. Assessment of vegetation and soil conditions in restored mangroves interrupted by severe tropical typhoon chan-hom in the philippines. Hydrobiologia.

Salmo, S., Lovelock, C. and Duke, N., 2013b. Vegetation and soil characteristics as indicators of restoration trajectories in restored mangroves. Hydrobiologia.

Schauber, E. M., Goodwin, B. J., Jones, C. G. and Ostfeld, R. S., 2007. Spatial selection and inheritance: Applying evolutionary concepts to population dynamics in heterogeneous space. Ecology 88, pp. 1112-1118.

Spalding, M., Kainuma, M. and Collins, L., 2010. World Atlas of Mangroves. Earthscan. 